\title{
Arbeitsgemeinschaften der DGU
}

\section{Urologe 2020 $59: 506$}

https://doi.org/10.1007/s00120-020-01170-4

Online publiziert: 11. März 2020

(c) Springer Medizin Verlag GmbH, ein Teil von Springer Nature 2020

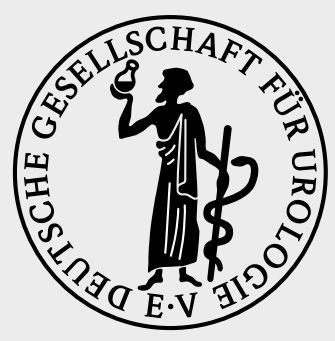

Herausgegeben vom Vorstand der Deutschen Gesellschaft für Urologie

Schriftführer

Prof. Dr. med. C. Wülfing, Hamburg

Schriftleitung

Rechtsanwalt F. Petersilie, LL.M. (V.i.S. d.P.)

Geschäftsstelle der Deutschen Gesellschaft für Urologie e.V.

Uerdinger Str. 64

40474 Düsseldorf

\section{Die Arbeitsgemeinschaft (AG) Ambulante Urologie stellt sich vor}

Die AG Ambulante Urologie in der DGU fördert den Austausch und die Vernetzung zwischen Praxis und Klinik. Sie koordiniert daher in erster Linie themenbezogene Aufgaben, die (nur) intersektoral zu lösen sind. Die AG hilft, den Status der Wissenschaftlichkeit in der ambulanten urologischen Versorgung zu festigen. Sie fördert Bestrebungen ambulant tätiger Urologen, wissenschaftliche Fragestellungen zu formulieren und zu erforschen, mit dem Ziel die ambulante Versorgungsqualität nachhaltig zu verbessern. Zugleich begleitet sie die ambulante Urologie in wissenschaftlichen Positionierungen, die durch die wissenschaftliche Fachgesellschaft (DGU) erarbeitet werden.

Die AG Ambulante Urologie identifiziert Herausforderungen für die Urologische Fachdisziplin im ambulanten Sektor zum Nutzen unserer Patienten. Hierbei steht der demographische Wandel im Fokus.

Enge Zusammenarbeit und intensiven Austausch sucht die AG Ambulante Urologie besonders mit den Arbeitskreisen (beispielhaft: Geriatrische Urologie, Versorgungsforschung, Qualität und Ökonomie, Hygiene und Infektiologie, Schmerztherapie/Supportivtherapie/ Lebensqualität/Palliativmedizin Onkologie)

Die AG Ambulante Urologie entwickelt Projekte, welche praxisnah und handhabbar die Arbeit der ambulant tätigen Urolog(inn)en unterstützen.

\section{Aktuelle Projekte}

ASV, Onkologie-Vereinbarung, Kognition und Alpha-Blocker, Rolle des mpMRT in der Primärdiagnostik und bei der Active-SurveillanceStrategie des Prostatakarzinoms, Hygienemanagement in der Urologischen Ambulanz, Antibiotic Stewardship und Vermeidung von Plastikmüll in der Praxis.

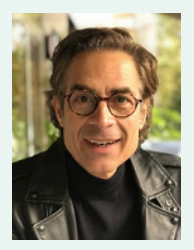

Vorsitzender: Dr. Thomas Speck (Berlin)

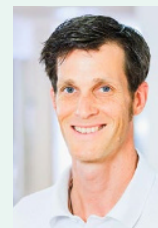

Stellv. Vorsitzender: PD Dr. Andreas Eisenhardt (Mülheim)

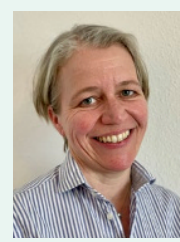

Schriftführerin: Dr. Kathrin Stein (Rostock)

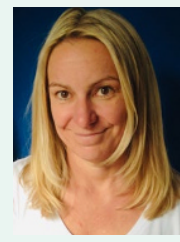

Dr. Annemie Loch (Sylt)

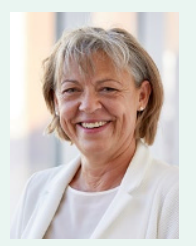

Franziska Engehausen (Berlin)

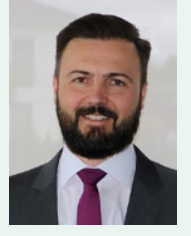

Dr. Frank Becker (Neunkirchen)

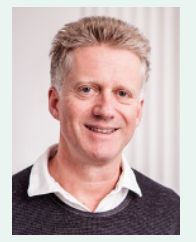

Prof. Dr. Michael Siebels (München)

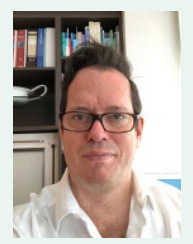

Dr. Philipp Spiegelhalder (Mettmann)

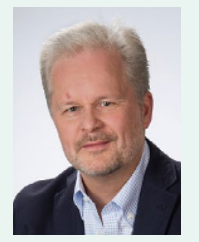

Dr. Michael Stephan-Odenthal (Leverkusen)

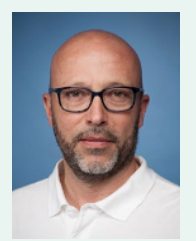

PD Dr. Frank König (Berlin) 